\title{
STXM_deconv - a MATLAB Script for the Deconvolution of STXM Images
}

\author{
Joshua Loroña Ornelas ${ }^{1}$, Benedikt Rösner ${ }^{2}$, Andreas Späth ${ }^{1}$ and Rainer H. Fink ${ }^{1,3, *}$ \\ 1. Physical Chemistry II, Friedrich-Alexander-Universität Erlangen-Nürnberg, Erlangen, Germany. \\ 2. Paul Scherrer Institut, 5232 Villigen PSI, Switzerland. \\ 3. CENEM, Friedrich-Alexander-Universität Erlangen-Nürnberg, Erlangen, Germany. \\ * Corresponding author, rainer.fink@fau.de
}

Over the last two decades scanning transmission X-ray microscopy (STXM) has experienced significant enhancements in terms of resolution. Improving spatial resolution in direct X-ray imaging is highly desirable to gain deeper understanding of many samples investigated with STXM. These enhanced resolutions were achieved by a constant improvement of the optics (most commonly Fresnel zone plates, FZPs) and the quality of the radiation source. Thereby resolutions have slowly been approaching the sub-10 nm regime [1, 2]. But pushing the experimental limits often means trade-offs regarding signal-tonoise ratios or image contrast. Therefore, post-processing the STXM micrographs can be useful to obtain improved image quality. It has been demonstrated long ago that post-processing by deconvolution is capable of improving the image quality of STXM images [3], but such processing has not found its way into common practice yet. One potential reason might be that the application of deconvolution algorithms to digital images can be difficult for users, who are not experienced in digital image processing or programming.

We present the program STXM_deconv for the restoration of STXM micrographs by deconvolution. It has been developed with focus on simplicity and accessibility for users without deeper knowledge on image processing. A graphical user interface (GUI) facilitates handling for users with limited programming experience.

The program was written in MATLAB version 9.2. MATLAB was used due to its good performance in processing numerical matrices, which also describe digital images. STXM images recorded in the NeXus HDF5 format [4] can be loaded into the program and saved as such. Furthermore, an import and export of ASCII text files is possible. For the deconvolution operation a point spread function (PSF) is required. This function is simulated with $S T X M$ deconv by an Airy pattern based on the illumination function taking into account the parameters of the utilized Fresnel zone plate and the photon energy. Alternatively, a previously generated file describing a PSF can be imported and can be optionally fitted with STXM_deconv. Two deconvolution algorithms common in image processing are presently implemented into STXM_deconv, i.e., the direct method of parametric Wiener filtering, where the user can change the intensity of the filtering by altering the noise-to-signal ratio (NSR) [5], and secondly a damped version of the iterative Lucy-Richardson algorithm, where the user can vary the number of iteration cycles and the damping parameter $\sigma$, which stands for the standard deviation of a compared Poisson distributed noise [6-8].

Examples of a highly resolved image processed with these two algorithms and a simulated PSF are shown in Fig. 1. The image shows a pattern of $9 \mathrm{~nm}$ lines and spaces. It was recorded at the PolLux beamline of the Swiss Light Source [9] with a Fresnel zone plate of $8.8 \mathrm{~nm}$ outermost zone width at a photon energy of $700 \mathrm{eV}$. The deconvolution operation alters the absolute values of the scale, but the quantitative information about the proportionality between objects of different transmittance is sustained. It is demonstrated that the deconvolution operations improve the image contrast and 
significantly damp the noise present in the original image, especially in the Wiener-filtered case. Also the resolution determined by Fourier shell correlation [10] gets enhanced. The resolution evaluated with the $1 / 2$ bit criterion is $7.9 \mathrm{~nm}$ for the original image and $5.1 \mathrm{~nm}$ for the example processed by the LucyRichardson algorithm with 15 iterations. Such restoration results will be improved further in upcoming program versions with addendums to $S T X M \_$deconv, e.g., additional algorithms [11].

The program and manual are made available at https://www.psi.ch/sls/pollux/analysis-tools

\section{References:}

[1] J Vila-Comamala et al, Ultramicroscopy 109 (2009) 1360-1364.

[2] B Rösner et al, Microelectron. Eng. 191 (2018) 91-96.

[3] C Jacobsen et al, Opt Comm 86 (1991) 351-356.

[4] B Watts and J Raabe, AIP Conf. Proc.1696 (2016) 020042.

[5] N Wiener, "Extrapolation, Interpolation, and Smoothing of Stationary Time Series: With Engineering Applications" (Technology Press of the Massachusetts Institute of Technology, Cambridge, Massachusetts)

[6] L B Lucy, Astron. J. 79 (1974) 745-754.

[7] W H Richardson, J. Opt. Soc. Am. 62 (1972) 55-59.

[8] R L White, The Restoration of HST Images and Spectra II (1994) 104-110.

[9] J Raabe et al, Rev. Sci. Instrum. 79 (2008) 113704.

[10] van Heel M and Schatz M, J Struct Biol 151 (2005), p. 250.

[11] We acknowledge funding by the German Minister of Education and Research (BMBF, contract 05 K16 WED), the EU-Horizon 2020 Research and Innovation Programme, No. 654360 NFFAEurope and the DFG within GRK 1896.
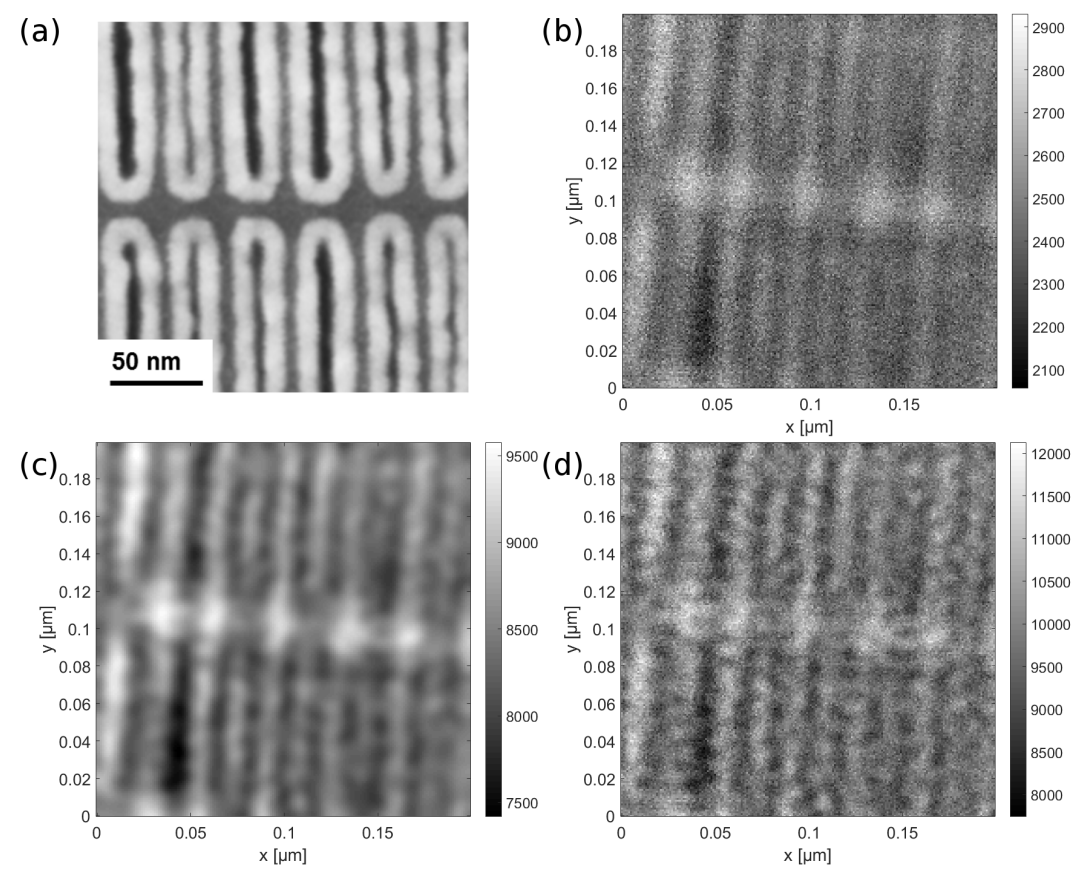

Figure. 1. (a) Representative SEM image of the test structure. It is scaled and $200 \times 200 \mathrm{~nm}$. (b) Original STXM image (not the same spot). It is $200 \times 200 \mathrm{~nm}$. (c) Wiener deconvolved image with an NSR of 0.01. (d) Lucy-Richardson deconvolved image with 15 iterations and a damping factor $\sigma$ of 0.05 . 